\title{
ODEWiki: A Semantic Wiki That Interoperates with the ODESeW Semantic Portal
}

\author{
Adrián Siles, Angel López-Cima, Oscar Corcho, and Asunción Gómez-Pérez \\ Facultad de Informática, Universidad Politécnica de Madrid, \\ Campus de Montegancedo, sn. 28660 Boadilla del Monte (Madrid, Spain) \\ asiles@delicias.dia.fi.upm.es, \{alopez, ocorcho, asun\}@fi.upm.es
}

\begin{abstract}
We present ODEWiki, a technology for the development of Semantic Wikis, which has a combined set of added-value features over other existing semantic wikis in the state of the art. Namely, ODEWiki interoperates with an existing semantic portal technology (ODESeW), it manages inconsistencies raised because of the distributed nature of knowledge base development and maintenance, it uses RDFa for the annotation of the resulting wiki pages, it follows a WYSIWYG approach, and it allows decoupling wiki pages and ontology instances, that is, a wiki page may contain one or several ontology instances. Although some of these features appear in some of the state-of-the-art semantic wikis, but they are not combined together in a single solution.
\end{abstract}

Keywords: Semantic Wiki, ODEWiki.

\section{Introduction and Background}

A wiki is software that allows users to create, edit, and link web pages easily ${ }^{1}$. Wikis are often used to create collaborative websites and to power community websites. As it happens with other Web sites, wikis are mainly focused on the provision of content for human users, and lack from a clear semantic description of their content. Semantic wikis have appeared in the last years as a technology that builds on top of existing wikis, providing the same functionalities as these plus the possibility of adding semantic annotations (normally in the form of RDF triples) to the wiki pages that are generated.

In the last years, there has been a huge growth in the number of semantic wikis that have been developed and made available to the community. For instance, the OntoWorld site provides a non-exhaustive list of approximately 30 semantic wikis ${ }^{2}$.In addition, there have been several workshops whose only topic has been about semantic wikis. In our analysis, we have focused on some of the most popular ones: Makna [1], Rhizome [2], Semantic Mediawiki [3], SweetWiki [4] and IkeWiki [5].

Some of the common characteristics of all these technologies are related to the following aspects:

\footnotetext{
${ }^{1}$ http://en.wikipedia.org/wiki/Wiki

${ }^{2}$ http://ontoworld.org/wiki/Semantic_Wiki_State_Of_The_Art
} 
- Annotation. This is the mechanism by which semantic annotations are related to the information provided in a wiki page (normally in a textual form). These semantic annotations may be related to existing ontologies or only be plain RDF, which is used mainly as a syntax for providing additional information.

- Edition. This is the mechanism by which users can add semantic information not only to the wiki pages that they are editing (this is the annotation process aforementioned), but also the knowledge base that acts as a knowledge repository for those wiki pages.

- Semantic search. This is the mechanism by which users may look for wiki pages taking into account their semantic annotations.

- Visualization. This is the mechanism by which semantic wikis visualize the semantic information about ontologies and their corresponding instances in the knowledge base.

- Navigation. This is the mechanism by which users can navigate between wiki pages taking into account the semantic relationships that are established by the annotations associated to them.

Although these are common features for semantic wikis, not all the existing semantic wiki technologies offer all these services, and there are many differences among different technologies with respect to the degree of complexity and functionality in each of these categories.

After an analysis of the state of the art in semantic wiki technologies, we have discovered several limitations of all of them:

- None of the existing semantic wikis is used together with a knowledge portal. This means that, although these applications are similar (semantic wikis and knowledge portals allow creating and maintaining knowledge bases), their strengths are not combined in any single solution. This could facilitate processes of collaborative knowledge update and different publishing options for knowledge portals, and knowledge edition and curation workflows for semantic wikis.

- Only one of the analyzed semantic wiki technologies (SweetWiki) makes use of the latest developments in the use of XHTML tags to include RDF triples $\left(\mathrm{RDFa}^{3}\right)$. This fact is very important for automatic data discovery using same wiki page, that is, persons and computers can be extract same information from this page.

- Only one of the analyzed semantic wiki technologies (SweetWiki) follows a WYSIWYG (What You See Is What You Get) approach, which facilitates the annotation of wiki pages for non-experts. The rest of technologies require users to learn a new set of tags or syntax to include these annotations, and also require users to know the ontologies to be used in advance, or to have them opened in a different part of the web site.

- In most cases, each wiki page is associated to only one instance of an ontology. This means that each page may only describe an individual, which makes it difficult, for instance, to create and annotate more complex pages which contain and declare several instances (e.g., a page that contains information about a set of persons that belong to an institution, where we want to have more information about them than simply their name).

\footnotetext{
${ }^{3}$ http://www.w3.org/TR/xhtml-rdfa-primer/
} 
Taking into account the aforementioned limitations, we have designed and implemented ODEWiki, which is a semantic wiki that is integrated with the ODESeW semantic portal [6] [7] [8]. This semantic wiki provides the most common functionalities that are provided by most of the existing semantic wikis: annotation, edition, search, navigation and visualization. And besides being integrated with a semantic portal, which acts as a knowledge repository for the wiki plus as an alternative knowledge publishing system. ODEWiki combines features that are only found in some of the state-of-the-art semantic wikis, such as the use of RDFa for the annotation of the resulting wiki pages, a WYSIWYG approach, and the decoupling of wiki pages and ontology instances, that is, a wiki page may contain one or several ontology instances.

In this demo, we will demonstrate how to edit semantically enabled wiki pages with this technology, together with the advantages of the integration with a semantic portal, so that the visitor will know how to operate with this technology.

\section{Annotation and Edition in ODEWiki}

Most of the limitations identified in the state of art are related to characteristics of the annotation and edition functionalities offered by wikis. In annotation and edition, users can use a WYSIWYG user interface to annotate wiki pages with semantic data, which is included in the wiki page using the RDFa format. This wiki page, and the semantic data included into it, is not modified until the user decides to modify it. This fact sometimes causes the semantic data to have external inconsistencies with the underlying knowledge base, and therefore with the ODESeW semantic portal or other wiki pages. ODEWiki provides a mechanism to automatically detect and manually correct these external inconsistencies. We understand by external inconsistencies those that are related to the following situations:

- The wiki page contains an instance that is not present in the knowledge base (e.g., "onto:AdriánSiles rdf:type onto:Person"). This may have happened because another wiki page or an external system has deleted that instance from the knowledge base.

- The wiki page contains annotations of an instance that exists in the knowledge base. However, the annotation itself (in other words, the RDF triple) does not exist in the knowledge base 4 (e.g. "onto:AdriánSiles onto:belongsTo onto:UPM", where onto:belongsTo has been removed from the ontology, onto:UPM has disappeared, this person has stopped belonging to onto:UPM, etc.). To some extent, this may be seen as a generalization of the previous case.

These external inconsistencies are checked whenever a wiki page is going to be visualized or when the user is going to start editing it. The user is notified of the

\footnotetext{
${ }^{4}$ Taking into account the Open World Assumption (OWA), this situation should not be considered as a problem, since we can always have annotations about an ontology instance that has not been defined elsewhere, but since this system is connected to a knowledge portal where we consider the Closed World Assumption (CWA), we must assume here this behavior. This behavior, however, could be changed accordingly in case that the OWA.
} 


\section{Full name: Ontology Engineering Group \\ Acronym: \\ Team is formed by: \\ - Adrian Siles (asiles@delicias.dia.fi.upm.es) \\ - Angel lopez (alopez@delicias.dia.fi.upm.es)}

Fig. 1. Notification of inconsistencies in a wiki page

inconsistencies that have been found (as shown in figure 1) and can take any of the following decisions: delete the corresponding RDF triple from the wiki page or from the knowledge base, or include the RDF triple into the knowledge base.

Users can annotate wiki pages in two ways, using semantic data from the knowledge base or adding new semantic data. Semantic data added to a wiki page is automatically added into the knowledge base. Not only can users add semantic data into the underlying knowledge base, but also can they delete, using a special action attribute in RDFa triple, semantic data form the knowledge base and from the wiki page. Users are responsible to take specific actions to avoid deleting relevant semantic data. In fact, ODEWiki is not recommended to be used in open environments, that is, ODEWiki is specifically designed to be used inside companies or organizations.

ODEWiki also provides a mechanism to automatically detect and manually correct internal inconsistencies. We understand by internal inconsistencies those that are related to the following situation:

A wiki page contains annotations of an instance that is, at the same time, removed from that page ("onto:AdriánSiles onto:belongsTo onto:UPM" is added to the knowledge base from the wiki page, but at the same time the wiki page contains an action to remove the triple "onto:AdriánSiles rdf:type onto:Person" and there is no other "onto:AdriánSiles rdf:type X" or "onto:AdriánSiles rdf:label X" triple in the knowledge base, which causes the removal of that instance).

\section{Conclusions and Future Work}

In this paper, we have shown some of the limitations of the most relevant semantic wiki technologies, which are mainly, related to their annotation and edition functionalities, especially in what respects to collaborative and distributed edition of semantic information. To overcome these limitations, we have built ODEWiki, a semantic wiki that is interconnected with a knowledge portal, which acts both as a knowledge repository and as an alternative publication and knowledge edition platform.

As part of our future work in this technology, we will focus on the provision of an improved search functionality that goes beyond the current state of the art in semantic search in existing semantic wikis (which is based on the SPARQL protocol). And in an improved navigation model that inherits some of the characteristics of the navigation model of ODESeW [8]. 


\section{Acknowledgement}

This work has been funded by the UPM-funded project "Extensiones a portales semánticos con wikis semántico", (CCG06-UPM/INF-284).

\section{References}

1. Nixon, L.J.B., Paslaru, E., Simperl, B.: Makna and multimakna: towards semantic and multimedia capability in wikis for the emerging web. In: Proceedings of the Semantics 2006, Vienna, Austria (November 2006)

2. Souzis, A.: Bringing the "wiki-way" to the semantic web with rhizome. In: Völkel, M., Schaert, S. (eds.) Proceedings of the First Workshop on Semantic Wikis From Wiki To Semantics, Workshop on SemanticWikis (ESWC2006), Budva, Montenegro (June 2006)

3. Millard, I., Jaffri, A., Glaser, H., Rodriguez-Castro, B.: Using a semantic mediawiki to interact with a knowledge based infrustructure. In: 15th International Conference on Knowledge Engineering and Knowledge Management (EKAW 2006), Podebrady, Czech Republic (October 2006)

4. Buffa, M., Gandon, F.: Sweetwiki: semantic web enabled technologies in wiki. In: Proceedings of the international symposium on Symposium on Wikis (WikiSym 2006), New York, NY, USA, pp. 69-78 (2006)

5. Schaffert, S.: Ikewiki: A semantic wiki for collaborative knowledge management. In: 1st International Workshop on Semantic Technologies in Collaborative Applications (STICA 2006), Manchester, UK (June 2006)

6. López-Cima, Á., de Figueroa, M.d.C.S., Gómez-Pérez, A.: The ODESeW platform as a tool for managing EU projects: the Knowledge Web case study. In: Managing Knowledge in a World of Networks. 15th Internation Conference (EKAW 2006), Podêbrady, Czech Republic (October 2006)

7. López-Cima, Á., Gómez-Pérez, A.: Rapid ontology-based Web application development with JSTL. In: Scripring for the Semantc Web 2007 from European Semantic Web Conference (ESWC 2007), Innsbruck, Austria (June 2007)

8. López-Cima, A., Corcho, O., Gómez-Pérez, A.: A Platform for the Development of Semantic Web portals. In: 6th International Conference on Web Engineering (ICWE 2006), Stanford, CA (July 2006)

\footnotetext{
${ }^{5}$ Extensions to semantic portals with semantic wikis.
} 